\title{
Novel Designs of Tin-Glass for Structural and Finishing Materials
}

\author{
V. Bugas ${ }^{1}$, M. Diakoumi, K. Gidado, E. Manzanares, E. Sazhina ${ }^{2}$
}

\begin{abstract}
Previously, we described the various environmental, commercial and social benefits from aesthetical available products, made from waste glass and concrete, which conform to the current sustainable composite materials' regulations in UK [3]. Moreover, we have reviewed [2] the state-of-the art research on composite materials made from recycled waste glass. Here, in this paper we focus on two novel designs of composite materials, first, a cube block, and, then, of control beams made from recycled tin-glass (TG). In cube blocks TG is placed inside its core, and in control beams TG is placed in three different orientations. There are two main categories of products which can be created by applying those two novel designs; structural materials and finishing materials. For structural materials, we propose a new method of placing a cover of metal on top of the TG when it is fully-immersed in the concrete. That avoids the contact of glass with the concrete which otherwise would start an Alkali silica Reaction (ASR). For finishing materials, we propose that one surface of TG is not completely covered with concrete, but it is allowed to be externally visible for main better aesthetical results. We describe in details the two novel designs of a cube block and control beams, and their application for structural and finishing materials.
\end{abstract}

Keywords: Tin-Glass, sustainable, Tin-Glass cube, Tin-Glass beam, structural design;

\subsection{Introduction}

The scope of this paper is to development of innovative solutions for waste glass into construction industry. Environmental benefits of waste glass products for building construction are explored with a particular emphasis on TinGlass construction unit following the patent filed in May 2008. The unit consists of a glass core encapsulated in a metal shell; it is produced by fusing glass cullet placed in a waste steel can such as baked. The glass cullet is provided by on-site granulation of various waste glass steams without need of segregation by colour. Thus two waste streams are processed together resulting in a construction unit of high mechanical compression strength. Current study looks into implementation of the TinGlass unit into a product for construction industry. The main focus will be on structural elements such as precast beams. On the other hand, a range of finishing materials such as glass bricks are designed and manufactured by the author. Both topics are seen as eco-alternatives to the traditional techniques.

\subsection{Structural elements}

Reinforcing concrete with TinGlass construction unit is perceived as the extension of traditional technique of reinforcing by steel rods. Both new and traditional techniques are subject to corrosion when exposed to air. This is not a problem when the reinforcing rod is buried with concrete [6], [7], hence the same attitude is accepted for the novel TinGlass reinforcement. Both techniques crucially depend on good bonding between steel and

\footnotetext{
11v.bugas@hotmail.co.uk

2 e.m.sazhina@brighton.ac.uk
} 
concrete. Whilst it is an established fact for conventional steel rods reinforcement, it must be explored for TinGlass reinforcement. This is an identified gap in knowledge and the study will be focusing on it. This topic has resulted in designing and manufacturing of TinGlass Concrete Beams (TGCB) for structural applications: An extensive experimental programme of designing, manufacturing TG's for testing in concrete beams and in concrete cubes. Analytical calculations and assessments are based on British Standards in compression [5] and test in bending [4]. The aim is seen in formulating recommendations for construction industry in using precast beams with TinGlass reinforcement. This is seen as a viable way of combating the alkali silica reaction (ASR). Other benefits include replacement of prime materials by the waste materials comprising TinGlass unit, and enhanced mechanical and thermal properties.

\subsection{Finishing materials}

Wall finishing by TinGlass bricks with exposed glass surface is an eco-alternative to flint wall technique. A number of prototypes of glass bricks, and a wall fragment, have been designed and manufactured by the author figures in Appendix. A technique for producing glass bricks without metal shell has been developed by the author as by-product of the work. Bricks of various colours and shapes (round, hollow, rectangular) are produced in a cheap and efficient manner.

\subsection{Background of research}

Recent research in the glass recycling workshop of School of Environment and Technology, University of Brighton, has resulted in a number of prototypes for cheap and energy efficient products made of waste glass.

A concept of TinGlass block originated in 2006 and patented in May 2008. This is the result of research work by $\mathrm{Mr} \mathrm{M}$. Bataineh in his MSc PID thesis [1]. The TinGlass construction unit is produced by fusing waste glass cullet in a waste steel can. Further development of this work by the author is in progress. The work has started with producing TinGlass blocks for incorporating them in beams for the needs of construction industry following the existing methodology of the patent.

In the course of this work, however, this research has developed its own methodology that had resulted in a number of novel glass brick prototypes.

\subsection{Modified TinGlass for structural purpose}

The scope of the production of a modified functional unit is that the TG had to be embedded in the concrete matrix to replace concrete mass. Using the proposed product of Mr M. Bataineh wasn't possible to imbed that functional unit because of the missing of the lid of the TG. After imbedding in the concrete, the original unit has a glass surface that could be in direct contact with the concrete that could be subject of ASR so it wasn't possible to use in such conditions in concrete.

A new design is prepared based in the referred patent and the new design is based on the needs to place the new product in concrete cubes and concrete beams. This product has to be applied in construction industry so the placing of the TG had to create the minimum number of modifications of an existed production - line of concrete beams. No wires are placed in the TG as this save time to prepare the TG and could not establish a standard characteristics of wires as most of them could bent during the transportation of the production. Hooks are placed on the two bases of the cylindrical shape which could present more constant mechanical characteristics and constant length. The operation needed more 
accuracy to bind the lid and the hooks. Such work needed more labour in the laboratory and a lot of changes of an existing line process to make. The increase of the time to prepare the TG's by one third from the non application of them forced to decide to not use the hooks.

The lid was placed on the top of the melted glass which bounded with that and created a barrier to the direct contact of the glass with concrete. The new product is easily pleased in a cube or beams as is not needed time to connect the wires in the beam and change the production process a lot. Just put a TG in the cube and place the TG's on the supports of the reinforcing rods of the beam. The process of producing TG concrete cubes is described later as shown in the next figures

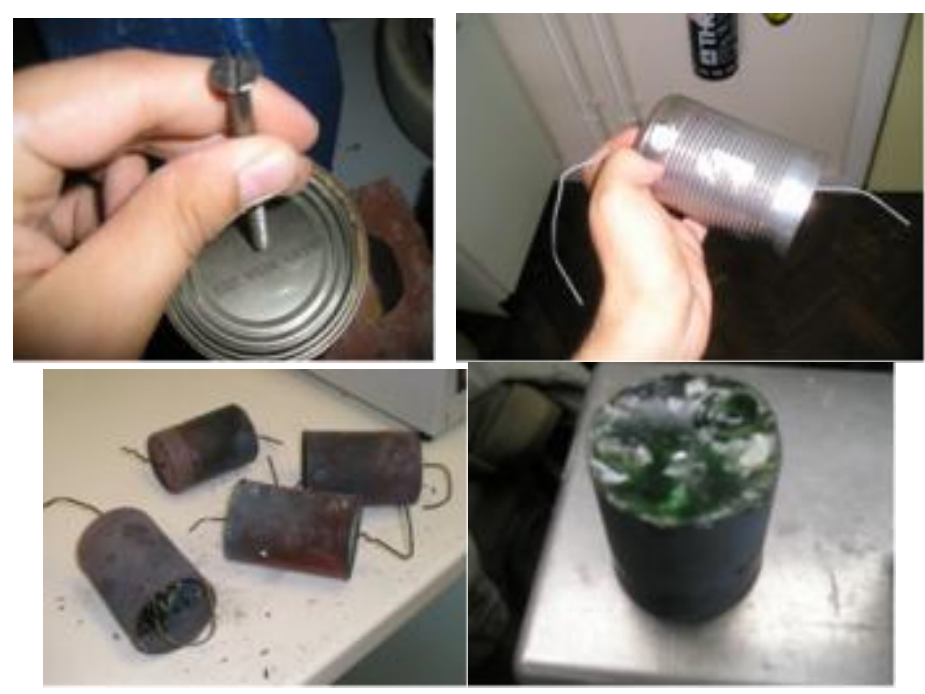

Fig. 1 Mr M. Bataineh's patent.

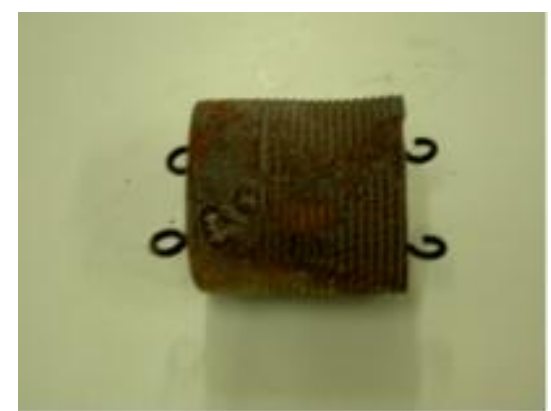

Fig. 2 Alternative to Mr M. Bataineh's made by the Author

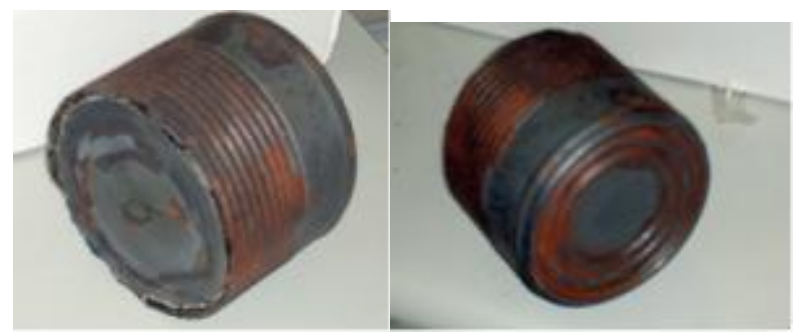

Fig.3 New final design of TG to apply in cubes and beams made by the Author

\subsection{Way of producing the new TG}


After granulate the available bottles and jags in the granulator in the first stages are used to put the glass in the tin cans without make any difference of size of grains. Then were placed in the oven and preheated for one hour at $300{ }^{\circ} \mathrm{C}$ created for not damaged the alumina of the oven. Then is increased the temperature at $950{ }^{\circ} \mathrm{C}$ and left for two hours. The glass was melting in the oven when curing for two hours at $950{ }^{\circ} \mathrm{C}$, and then left cooling in the oven for ten hours was presenting a smooth surface. The TinGlass embedded in concrete in such way that concrete surround only the metallic part of the TinGlass is created the alternative of flint wall.

Different time of curing and different size of grants make tin glasses not have horizontal surface. This because of the different time that needed to be melted the small and the bigger grains. The result is presented in the Fig.4 there for is decide to sieve the glass to place layers with the same size of the grains and have an horizontal position of the lid after as final form of the TinGlass uniform.

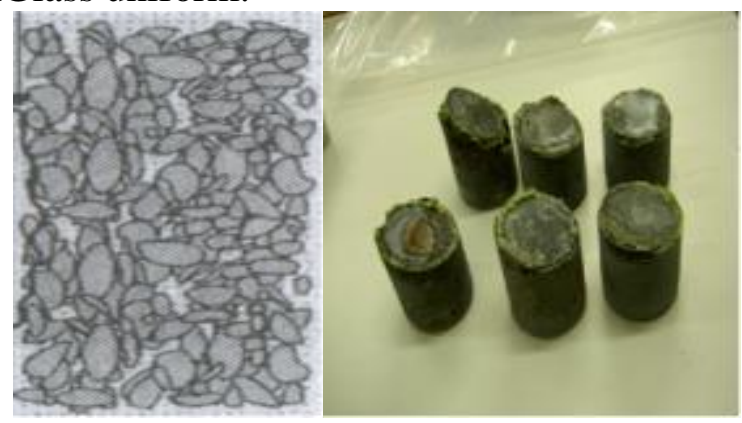

Fig.4. The bigger grains of the glass were melting later than the smaller and the surface of the lid weren't horizontal as there are created gaps in the process of melting.

This problem presented when for safety reasons of the production of the TG's with lid is decide to put the lid in the initial stage of baking the TG's. In case of putting the lid after one and half hour in the oven at $950^{\circ} \mathrm{C}$ baked the glass was melted and the lid appeared horizontal at the end of the process.

A new technique is developed in the laboratory of UoB to create TG's with horizontal lids to satisfy (Fig.3) the needs of the new composite product. A lid is placed over the glass insert while the glass is still warm. As the glass cools, the lid may become adhered to the solidified insert of glass or for safety reasons is placed from the begging before put the tin can with the glass culet in the oven. The results were good but still the problem of the safety to place a lid at $950^{\circ} \mathrm{C}$ existed so is designed a cylindrical dick of $150 \mathrm{gr}$ of weight and diameter $67 \mathrm{~mm}$ to be placed on the top of the lid (Fig. 5). The stages of the preparation of TG's before placed in the oven are presented in the fig. 6

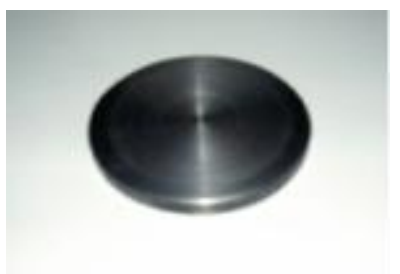

Fig. 5.Cylindrical dick of $150 \mathrm{gr}$ of weight and diameter $67 \mathrm{~mm}$ t placed on the top of the lid 

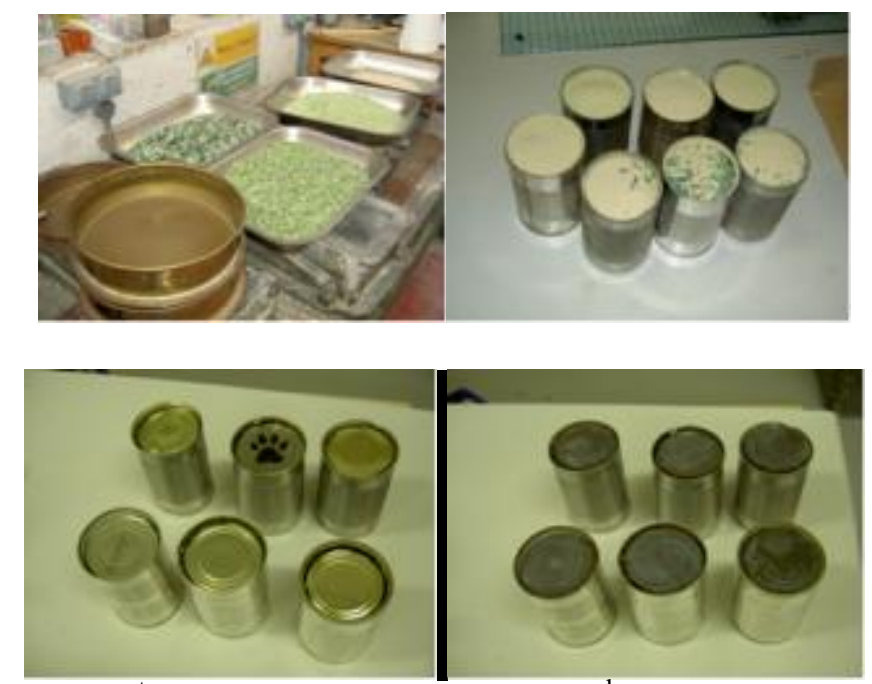

Fig.6. Stages of RG's $1^{\text {st }}$ sieved granulated glass, $2^{\text {nd }}$ after placed the tin can, $3^{\text {rd }}$ after placed the lid, $4^{\text {th }}$ after placed the appropriate weight on the top of the lid before put in the oven.

Thus the problem is solved by putting small lays of the same size of grains in the Tin Can and very fine grains under the lids as shown in fig 6 in this way the very fine glass when is melting is sticking with the lid after placed the appropriate weight on the top of the lid and is this way the lid is keeping horizontal. In these embodiments, the shell therefore acts as a permanent mould. The orientation of the lid is horizontal that satisfy the dimensions of the TG for the composite cubes and beams as in fig 5 .

\subsection{Embedding TG in concrete for structural purpose}

This part of research is focused on developing an innovative composite material by embedding Tin-Glass (TG) unit in concrete to form the Tin Glass Concrete Unit (TGCU) for structural purpose. The inclusion of the TG units provides a way of reusing glass and other waste materials e.g. metal, reducing the need to use non-renewable materials, and increasing levels of recycling, which is increasingly important in the construction industry. Thus, it is believed that the embedding of the TG units in concrete is advantageous in its own right for environmental reasons.

The mechanical properties of glass and concrete should be close to conventional materials commonly used in structures. To investigate any difference in the structural behaviour of TG composite material in relation to conventional concrete units, tests in progress in the laboratory. The concrete mixture and sampling have been prepared according to standard specifications (BS 1881-125:1986) adopted by the construction industry.

The synthesis of the concrete mixture comprised of water, cement, sand and aggregate. The ratio of water: cement: sand: aggregate used in the mixture was 0.6-1-2-4 conforming to BS [6] and was mixed in the laboratory.

The type of cement used was Portland cement and the sand was natural composed of grain sizes given in Table 1. The coarse aggregates were local crushed aggregates from a river bed with a maximum diameter of $100 \mathrm{~mm}$ and a semi-rounded shape 


\begin{tabular}{|l|l|l|}
\hline Sieve size [mm] & $\begin{array}{l}\text { A1 Quantity of aggregate } \\
\mathbf{1 0 4 9} \text { [gr] }\end{array}$ & $\begin{array}{l}\text { A2 Quantity of aggregate } \\
\mathbf{1 0 4 0} \text { [gr] }\end{array}$ \\
\hline 2.36 & 174 & 168 \\
\hline 2.00 & 25 & 25 \\
\hline 1.18 & 54 & 54 \\
\hline 0.71 & 44 & 45 \\
\hline 0.60 & 15 & 15 \\
\hline 0.30 & 161 & 173 \\
\hline 0.25 & 140 & 132 \\
\hline 0.15 & 431 & 424 \\
\hline & 5 & 6 \\
\hline
\end{tabular}

Table 1: Results obtained from the sieving test of sand.

\subsection{Description of the design Tin Glass concrete cube}

The dimensions, for casting of the cubes are specified by the following reference, BS [4]. The internal dimensions of each mould in BS standard are 10X10X10 cm3.

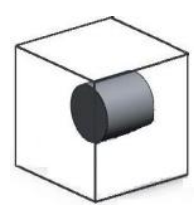

Fig.7.TG construction cube

The volume of the TinGass, placed in the mould, is $270 \mathrm{~cm} 3$; as the dimensions of the TGCU are: a diameter of $7 \mathrm{~cm}$ and a height of $7 \mathrm{~cm}$. After placed the TGCU in the core of cube the distance of the TGCU from all surfaces of the cube is $1.5 \mathrm{~cm}$ as shown in fig. 7 fill the rest of the mould with concrete.

\subsection{Description of the design of TGMC beams}

The scope of the new design is to replace prime materials comprising concrete with TGCU which can be perceived as an extension of the traditional technique. Hence the TGCB were produced by placing the TG units along the neutral axis of the beam TinGlass are to be placed on the neutral axis of the beam; hence, the centre weigh point does not change the uniform mass of the TinGlasses. What is to be expected is that this will not affect the behaviour of the beam on the compression and tension area.

The TG units were placed in the beam in three different orientations: the longitudinal axis of the TG unit coincided with coincided with is the longitudinal axis of the beam the longitudinal axis of the TG unit coincided with the transverse axis of the beam, the longitudinal axis of the TG unit coincided with the vertical axis of the beam. The dimensions of all moulds used were the same and equal to $0.71 \mathrm{~m}$ by $0.15 \mathrm{~m}$ by $0.15 \mathrm{~m}$ the shape and dimensions of the moulds agree with the BS [4] specifications.

The TGCBs had approximately similar weight with the control beams. This can be explained by the fact that the density of the concrete is $2.3 \mathrm{e} 3-2.6 \mathrm{e} 3 \mathrm{~kg} / \mathrm{m} 3$ close to the density of Soda-lime glass is: $2.44 \mathrm{e} 3-2.49 \mathrm{e} 3 \mathrm{~kg} / \mathrm{m} 3$, as reported in the CES EduPack [8]. The dimensions of the TinGlass as a construction unit are designed in such way as to be $7 \mathrm{~cm}$ in height and $7 \mathrm{~cm}$ in diameter. As an example of this, the weight of a given batch, before testing the control beam was $36.8 \mathrm{~kg}$ and the other two composite beams was 38.0 $\mathrm{kg}$ and $37.5 \mathrm{~kg}$ respectively. 
The concept of a neutral axis is defined here as the location of zero bending stresses in a beam. The neutral axis passes through the centroid of a cross-section, and the bending stresses in concrete are develop linearly with distance. [9]. This is a realistic assumption for the elastic analysis of the beam before the appearance of any cracks. After that, the reinforcing rods in the tensile part of the beam hold the concrete together.

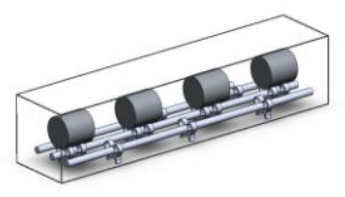

Orientation A

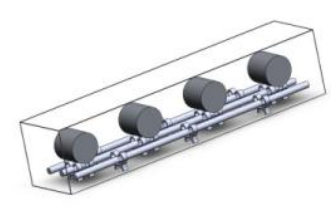

Orientation B

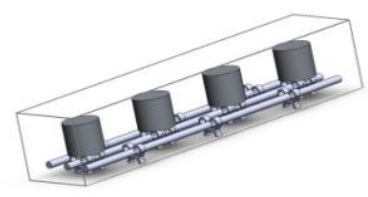

Orientation C

Fig. 8. Cylindrical TinGlass blocks, embedded into a concrete beams

It is advanced that the distances of the TinGlasses when the orientation is changed but not the number or height of the TG's; it is important to compare the control with the TGCB's from the same batch. Further to this that the standard reinforcement of each beam is provided by three steel rods, in all cases control and TGCB.

The TGCB have a number of TinGlasses embedded into the beam at the casting stage. The TinGlass units are positioned above the steel rods, using additional plastic spacers placed in the marked positions. The neutral axis of the TinGlass is parallel to the $\mathrm{x}$ axis, longitudinal to the $\mathrm{x}$ axis and vertical to the $\mathrm{x}$ axis of the beam. Since the TG are placed at the centre of the cross section of the beam since the TG are placed at the centre of the cross section of the beam What this entails is that during the test in bending of the TGCB the areas that have been created to produce a concentration of stress that make the beams weaker should be more uniform around the symmetric distribution of the TG's on the neutral axis.

\subsection{Different products under the form of finishing materials}

A methodology for producing glass bricks from waste glass have been developed by the author with a view of decorative, practical. A range of attractive glass bricks and wall finishes emulating conventional flint walls is designed and manufactured by the author. 
Whilst the main focus of current research is seen as focusing on pre-cast beams with embedded TinGlass construction units.

\subsection{TinGlass surface finish as an eco-friendly alternative to flint wall}

The first prototypes produced by the author were based on existing methodology for the TinGlass. Melting and fusing of waste glass cullet in a tin-plated steel waste can is performed at $900^{\circ} \mathrm{C}$ for soaking time $40 \mathrm{~min}$. Subsequent annealing is performed by gradually reducing temperature to $500^{\circ} \mathrm{C}$ during two hours, then, without opening the kiln, switching off the power and leaving it overnight .

The novelty of approach by the author consists in applying this concept for creating wall finish seen as an eco-friendly alternative to conventional technique of flint walls. A TinGlass block is produced with top surface of glass remaining exposed. It is embedded into mortar mixture thus emulating the flint wall surface.

The finished wall is a composite material made from concrete and recycled glass. TinGlass bricks are placed in the concrete leaving a glass visible. It can be compared with flint wall without using natural stone but recycled glass instead.

A fragment of the wall finish is designed and manufactured in the workshops of SET. The boundary between glass and mortar on the wall surface can be either exposed, with rough edges of TinGlass brick protruding similar to a flint wall surface (see Fig.1a and Fig.1b in appendix photo of $\mathrm{Mr} \mathrm{H}$ Hills, SET). On other hand, for a flush wall finish, removing rough ends of TinGlass by polishing them off with a metal file, will achieve this aim

This product can be used for a variety of applications, including garden walls, vehicle crush barriers, flood or sea defences. It combines high density and mechanical strength and toughness with an attractive surface finish, allowing creation of artistic patterns and novel architectural features.

\subsection{Surface smoothness, opaque appearance and density of TinGlass construction unit}

In the course of extensive experimentation with various production regimes for TinGlass bricks it has been established by the author, that significant variations in surface roughness and density of the Tinglass unit can be achieved by varying length of soaking time in the kiln. Under the standard regime of soaking time $40 \mathrm{~min}$ at $900{ }^{\circ} \mathrm{C}$, the surface of the fused glass is rough. (See Fig. 2 and Fig. 3 in appendix) Increasing soaking time up to 2 hours at this temperature will create a smooth opaque surface and reduced volume of glass core (hence higher density).

This is because glass granules have melted under this regime rather than fused at $40 \mathrm{~min}$ soaking time. Heat transfer inside glass core is by heat conduction, and longer soaking times are raising temperatures inside glass cullet. Sufficient energy is supplied for glass phase change from solid to liquid state. TinGlass resembles a natural stone under this production regime. This is desired result for some of the applications where higher density and enhanced mechanical toughness is needed.

It has been observed by the author that the size of glass cullet granules bears much influence on the appearance of the final product. Large size of glass cullet granules gives a brighter and more translucent appearance to TinGlass than a glass powder.

\subsection{Aluminium cans as a mould absorbed by glass during fusing}

When fusing glass at high temperatures it was previously considered impossible to use an aluminium can (e.g. sardines can) as a mould since melting temperature of aluminium is 
lower than that of glass. Indeed earlier tests have shown a disintegration of aluminium mould and spilling molten glass in the kiln.

The author has developed a way to produce a waste glass brick or paving stone, with a mould made of sardines' can, by establishing the range of temperatures and soaking times for this. A paving stone made of waste glass by fusing it in a sardines can, is shown in Fig.6 in appendix. It was melted at $800^{\circ} \mathrm{C}$ with 1 hour soaking time and subsequent annealing overnight. On cooling, it was discovered that the aluminium mould is absorbed by the glass core, producing an attractive surface finish as the result.

This method can be used for production of paving stones with opaque surface with high coefficient of friction.

\subsection{Glass bricks without a permanent mould}

All cases described above had a metal can, whether steel or aluminium can, as a stay-on permanent mould. This section describes a method of production of waste glass bricks by extracting them from a mould on cooling.

Fusing or melting glass in a metal mould creates a very strong bond between them, and it is practically impossible to extract the glass core out of the mould on cooling. This is how the TinGlass concept has originated.

It is possible however to line the mould by ceramic fibre paper prior to heating. This greatly facilitates the extraction of glass core from the mould after annealing and cooling to room temperature. This method was advised by Mr D Watson [10], a local artist in glass, during his visit in summer 2007.

This method has been further developed by the author. He has succeeded in producing glass bricks of rectangular or round shape. Actually any reasonable shape can be produced by manufacturing a steel mould of given shape and lining it up with ceramic fibre paper.

The cross-section shape of the brick may be solid or hollow, with a central hole produced by insertion of a steel cylinder wrapped into ceramic fibre paper (see Fig.7, Fig.8 and Fig.9 in appendix). Hollow bricks may be incorporated into various structures by stacking them on a steel pole or similar support.

Sharp edges and corners, combined with matt and grainy surfaces in the areas lined by ceramic fibre paper, present a certain problem for the glass bricks produced by this method. The author has addressed this problem by testing and implementing a normalising method for glass bricks.

Description of normalizing method: After cooling glass bricks to room temperature and extracting them from the mould, re-heating and re-melting of surface layers is performed by placing the brick into a very hot kiln $\left(900^{\circ} \mathrm{C}\right)$ for 30 mins of soaking time, then annealing it overnight. This procedure makes the surface much smoother and gives it more shiny appearance than before.

\subsection{Conclusions}

This work aims to outline the potential of new design of innovative products which described above in order to use them as construction and finishing materials in the construction industry. 
Problems obtained during the production of TG and the position of the lid on the top of the TG are solved by using the same dimension of grains and putting a cylindrical dick of $150 \mathrm{gr}$ of weight and diameter $67 \mathrm{~mm}$ on the top of the lid.

Making a new product which is composed from a recyclable material and increase the life span of a recyclable material which is still recyclable has much potential as a vehicle for economic prosperity and creativity, because we are saving prime materials by creating new products.

\section{References}

[1] Bataineh, A.M., 2006 Innovative recycling and cooling solutions. Thesis University of Brigthton UK.

[2] Bugas, V. Afentouli, L. Manzanares, E. Sazhina, E. Harder, M.,2008. Producing wealth from waste glass: ecological, commercial and social dimension, SB08 MED International conference on Sustainable Building10, 11 and 12 January 2008, Athens, Greece.

[3] Bugas, V. Gidado, K. Manzanares, E. Pearce, D. Sazhina, E., 2009. Recycling Glass as Construction Material, SET FOR BRITAIN Presenters and posters Engineering 9 March 2009 London, UK.

[4] BS EN 12390-1:2001 Testing hardened Concrete. Shape, dimensions and other requirements for specimens and moulds

[5]BS EN 12390-3:2002 Testing hardened concrete. Compressive strength of test specimens

[6] BS1881-125:1986 Testing concrete. Methods for mixing and sampling fresh concrete in the laboratory

[7] B.S. 8110: Part 1:1985 Structural use of Concrete

[8] CES Selector ${ }^{\mathrm{TM}}$ software, Granta Design Lid, 2010 www.grantadesign.com, Cambridge UK [Accessed 11/02/12]

[9] Gere, G. (2001) Mechanics of Materials 5th edition: Brooks/Cole USA ISBN 053437133-7

[10]Watson, D.http://www.basingstoke.gov.uk/NR/rdonlyres/AC40DA7F-E2F3-44A398A5-B099FD79A69E/0/public_art_brochure.pdf [Accessed 21 June 08] 


\section{Appendix 1. Finishing materials for construction industry}

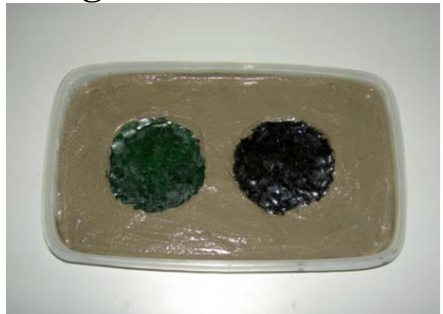

Fig1a. Fragment of wall finish with TinGlass units. This is seen as a take on the traditional flint wall technique as shown in Fig $1 b$.

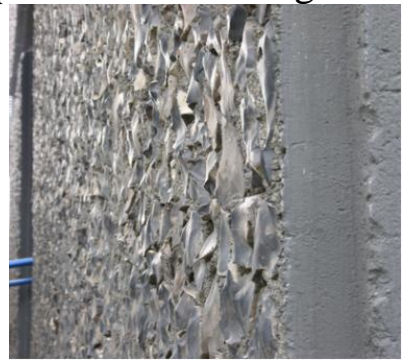

Fig.1b. Flint wall, Cockroft building, University of Brighton (UoB).

Photo taken by Mr H. Hills, SET, UoB

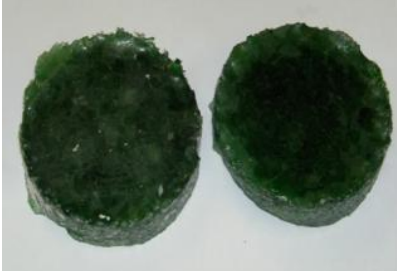

Fig.2 Opaque glass brick without metal shell

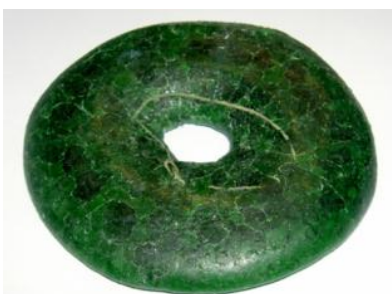

Fig.3 Opaque glass brick smoothed by normalizing (additional heating process)

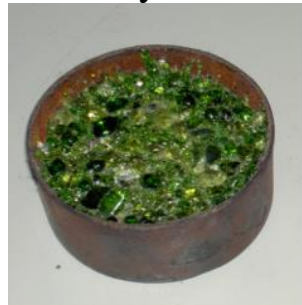

Fig. 4 Non-smooth surface of TinGlass unit produced by fusing glass cullet

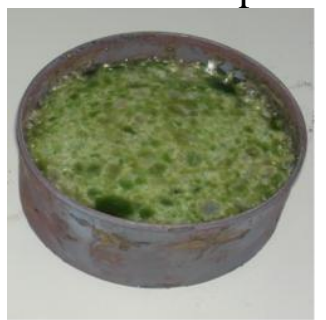

Fig 5. Smooth surface of TinGlass unit produced by melting glass cullet 


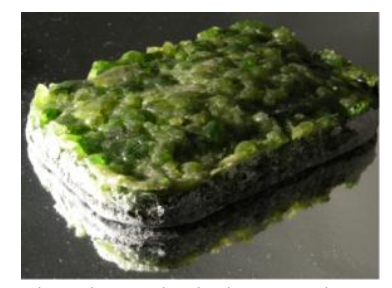

Fig. 6. Rectangular non-smooth glass brick produced by fusing in a sardines' can mould (Photo taken by Mr H. Hills, SET, UoB)

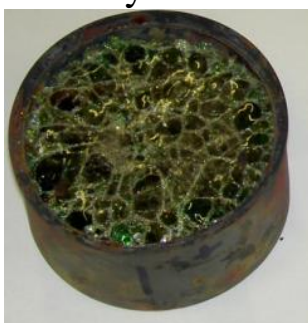

Fig 7.Thermal craze of TinGlass produced by quenching in water.

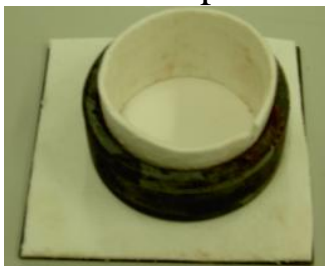

Fig. 8 Metal mould lined with ceramic paper prior to fill by glass cullet

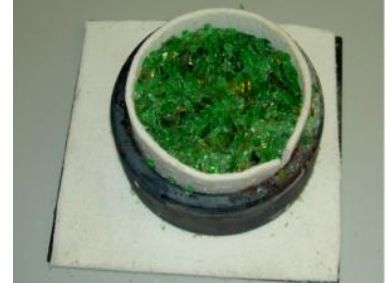

Fig.9 Glass cullet in round steel mould is prepared for the furnace

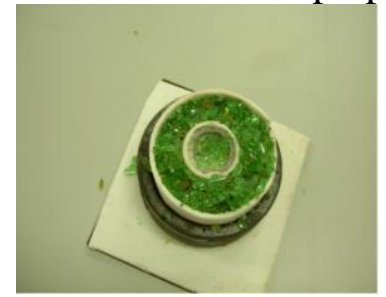

Fig.10 Hollow mould with glass cullet is prepared for placing in the furnace

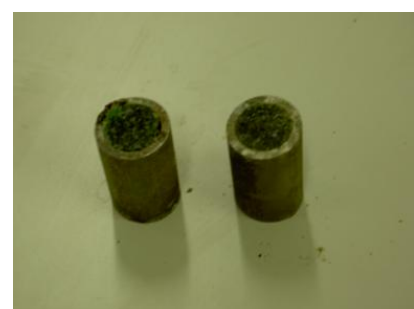

Fig. 10 metallic tubes designated to be placed to walls with a light on the back of the glass surface 